\title{
A New Deterministic Spatio-Temporal Continuum Model for Biofilm Development
}

\author{
HERMANN J. EBERL ${ }^{a, c, *}$, DAVID F. PARKER ${ }^{b}$ and MARK C.M. VAN LOOSDRECHT \\ ${ }^{\mathrm{a}}$ GSF National Research Center for Environment and Health Inst. Biomathematics and Biometry, 85764 Neuherberg, Germany, ${ }^{\mathrm{b}}$ Dept. \\ Mathematics and Statistics, University of Edinburgh, EH9 3JZ, UK and ${ }^{\complement}$ Dept. Biochemical Engineering, Delft UT, 2628BC Delft, The \\ Netherlands
}

(Received 22 March 2000; In final form 28 July 2000)

\begin{abstract}
A new mathematical model for the development of spatially heterogeneous biofilm structures is presented. Unlike previous hybrid discrete/continuum models it is a continuum model throughout, describing the interaction of nutrient availability and biomass production. Spatial biomass spreading is described by a nonlinear density-dependent diffusion mechanism. The diffusion operator degenerates for small biomass densities and is singular at the biomass density bound. The model can be interpreted as a predator-prey model for biomass and nutrients. First numerical simulations show that the model is able to predict experimentally observed cluster-and-channel biofilm structures. The results are reliable and in qualitatively good agreement with experimental expectations.
\end{abstract}

Keywords: biofilms, spatio-temporal mathematical modelling, density-dependent diffusion, numerical experiments

\section{INTRODUCTION - SPATIAL MODELLING OF BIOFILM PROCESSES}

\subsection{Definition, Occurrence, and Impact of Biofilms}

Biofilms are accumulations of micro-organisms growing on phase interfaces, embedded in a polymeric matrix. In this slime layer, other bacteria can be captured and a vivid microbial community develops in microcolonies. In biofilms, bacteria live in a protected mode of growth and this enhances their ability to survive in hostile environments. Consequently, when biofilms are involved in bacterial infections, they cannot be treated easily by antibiotic therapies (Costerton et al, 1999).

Biofilms play an important role in medicine. They cause microbial infections in the body, amongst them infections of airways and lungs, middle ear, oral soft tissues, gastrointestinal and urogenital tracts. Prostheses and implants (Kayser et al., 1993) like hip replacements, pacemakers, catheters, and artificial heart valves, as well as dead tissue, are susceptible to colonization by biofilms, which can lead to bacterial infections. A list of human infections involving bacterial biofilms is given in Costerton et al (1999). In the mouth, bacteria bind to proteins covering dental

\footnotetext{
* Corresponding Author.
} 
enamel, so leading to the development of dental plaque (Kayser et al., 1993). However, since biofilms grow wherever dissolved nutrients are available for feeding the micro-organisms, health risks from them are not restricted to colonizations in the body. For example, biofilms developing in water distribution systems or in kitchen sinks can cause problems of hygiene.

Sessile bacteria living within a biofilm colony grow under different conditions from those growing under planktonic conditions and so they behave differently. This is especially true in the interior of the biofilm, where nutrients and oxygen are limited. The bacteria there live in a slow-growing or starving mode, while they may be well-fed in the outer regions of the biofilm. Therefore, bacteria of the same species can develop very different metabolic states within one biofilm colony. Micro-organism response to local concentrations and concentration gradients is quantitatively very different in the two regimes of planktonic growth and of biofilms. For example, biofilm bacteria can withstand host immune reponses and they turn out to be more resistant to antimicrobial agents than their nonattached planktonic counterpart. Antibiotics may not be able to penetrate through the outer layer of bacteria and, therefore, may not reach the inner organisms, so that these can survive and multiply. This induces difficulties in medical treatment of biofilms settling in the human body. The cells cover a wide range of states and conditions, and, thus, allow at least some of them to survive any metabolic attack (Costerton et al. 1999). For a more extended overview of the role of bacterial biofilms in medicine, see Costerton et al. (1999) and the references cited therein.

The understanding of biofilm formation is important for devising medical treatment and for the prevention of biofilm-borne infections. Because of the distinctive behaviour of biofilm communities, they must be studied separately from planktonic bacteria. Biofilms form in many different environments and under very different conditions, therefore, no standard biofilm exists and the generality of experimental studies always suffers from the environmental conditions in the laboratory reactor and particular properties of the bacteria involved. We hope that mathematical modelling of biofilm processes on a very general and basic level will help towards their understanding them better. Therefore, we present a new mathematical approach for the development of spatially irregular biofilm structures.

\subsection{Recent Mathematical Models for Heterogeneous Biofilm Structures}

Mathematical models for biofilm processes have been formulated since the 1970s and 1980s (e.g. Rittmann \& McCarty, 1980, Kissel et al., 1984, Wanner \& Gujer, 1986, and the review of Chaudhry \& Beg, 1998). These first models were ordinary or one-dimensional partial differential equations assuming a biofilm which develops as a flat layer. Direct observations and new microscopy technologies, however, revealed that in realiter biofilms grow in highly irregular spatial structures (e.g. Gjaltema et al., 1994, or the review of Costerton et al., 1995) and, hence, that the assumption of flat layered biofilms was a gross simplification. Subsequently, multi-dimensional models capable of describing spatial non-uniformities have been developed in recent years, in addition to one-dimensional biofilm models which still are a useful tool for the global analysis of complex biological interactions when no local resolution is required.

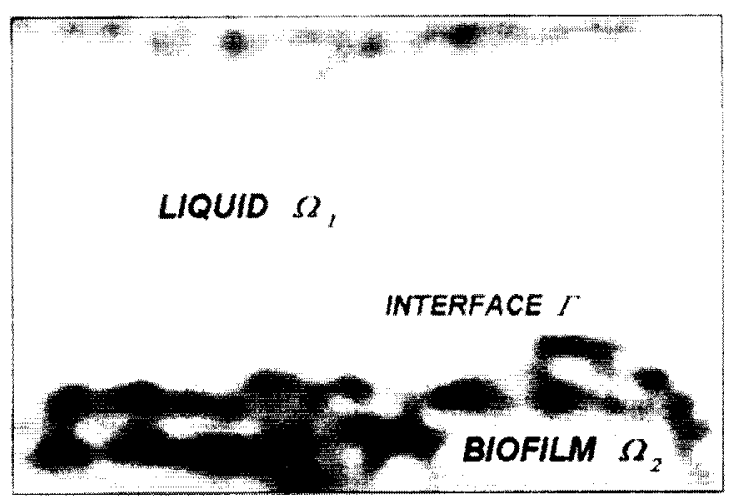

FIGURE 1 The computational domain $\Omega$ consists of a liquid region $\Omega_{1}$ and solid biofilm region $\Omega_{2}$ separated by an interface $\Gamma$ 
The multi-dimensional models have made an explicit distinction between a bulk liquid region $\Omega_{1}$ without biomass and a solid biofilm region $\Omega_{2}$ where all biomass is contained, as sketched in Figure 1. Thus, pores and channels in the biofilm filled with liquid belong to $\Omega_{1}$ according to this definition. Both regions are treated as continua separated by an interface $\Gamma$. In the solid region, nutrients are consumed in biochemical reactions and transported by a diffusion mechanism. In the liquid region, the transport of nutrients is due to diffusion and convection. An analysis of characteristic time scales of biofilm processes shows, that consumption of nutrients, and diffusive and convective transport are much faster than those processes governing the development of the biofilm structure. Following Kissel, 1984, it is therefore possible to decouple processes and to consider pseudo-steady state solutions of the more rapid processes. This was used in recent two- and three-dimensional steady state studies of the influence of spatial heterogeneities on nutrient transfer and consumption in complicated biofilm geometries by Rittmann et al (1999) (2D, cylindrical coordinates, diffusive transport), Picioreanu et al (2000) (2D, Cartesian coordinates, convective and diffusive transport), and Eberl et al. (in press) (3D, Cartesian coordinates, convective and diffusive transport).

The equations describing hydrodynamics, transport and consumption of nutrients, and biomass production are well established. Only little is known about modelling the actual mechanisms of biomass spreading and, hence, of formation of biofilm structures. It is influenced by many different biological, chemical, and physical factors. From numerous experimental studies, it is concluded that the shape in which a biofilm develops depends primarily on physical and environmental conditions (van Loosdrecht et al., 1995, 1997): Increased shear or detachment forces will lead to a smoother biofilm surface. The nutrient availability at the biofilm interface influences the local biofilm growth. In the case of a strong nutrient concentration gradient (due to fast consumption in $\Omega_{2}$ or low mass transport rates from $\Omega_{1}$ into $\Omega_{2}$ ), local variations are enhanced and a rough biofilm develops (Picioreanu et al., 1998b). The latter phenomenon occurs also in crystal growth and is as such well modelled. In the case of biofilms, however, new biomass is formed within the structure. This feature requires extra attention during model formulation. Several authors (Wimpenny \& Colosanti, 1997, Picioreanu et al, 1998a, Hermanowicz, 1999, Noguera et al., 1999) suggested a lattice discretization of the computational domain and use of spreading mechanisms according to a set of probabilistic, discrete, local rules: If the biomass density in a lattice cell exceeds or approaches a critical maximum value, either a specified or a random amount of biomass is transfered to a neighbouring grid cell. The selection of the new location is random. If there are no empty neighbours, different strategies can be applied to find an appropriate neighbour. It could be shown, that predictions of these probabilistic black boxes are qualitatively in good agreement with experimental expectations. However, these mathematical models have serious physical drawbacks:

- they are strongly lattice-dependent and, hence, they are not invariant to changes of the coordinate system

- it can be shown that local grid refinement will lead to different model outputs; in particular, local symmetry cannot always be obtained under symmetric environmental conditions.

- an ordering of grid cells must be specified before implementing the biomass spreading procedure, in order to avoid conflict when two grid cells try to move biomass onto a shared neighbour cell.

- the biomass, though a continuous variable according to growth kinetics, suffers discrete changes during splitting

- many possibilities exist for formulating local spreading rules which are apparently reliable but qualitatively different and, hence, they are somewhat arbitrary and might lead to aesthetically driven, rather than to physically motivated, model formulation.

Since all these issues arise from the discreteness of the spreading mechanism, it appears worthwhile to seek a fully continuum model, as an alternative. 


\section{A NEW CONTINUUM MODEL FOR BIOFILM GROWTH}

\subsection{Important Model Features}

A few properties are postulated in order that the model accords with experimental observation and with previous modelling results. These are

i. existence of a "sharp front" of biomass at the fluid/solid transition

ii. biomass spreading is significant only if a certain maximum density is approached

iii. biomass density can not exceed that maximum bound

iv. biomass production is due to standard reaction kinetics

v. the biomass spreading mechanism should be compatible with hydrodynamics and with nutrient transfer/consumption models

vi. for given biochemical parameters, spatial heterogeneities in a mono-species biofilm structure are due only to environmental conditions such as nutrient availability and initial or boundary conditions

From $i v$ ) it is concluded that the biomass bound iii) can not arise from the reaction terms, as in many other models of mathematical biology, but must be associated with the biomass spreading process itself. Furthermore, it can be hoped that it is an immediate consequence of $i i$ ).

Probably the first idea for formulating a continuum model with a sharp front behaviour is a convective transport mechanism for biomass. This was suggested in a one-dimensional model by Wanner \& Gujer (1986). In a recent paper, Wood \& Whittaker (1999) also followed this approach. In their approach, however, an evolution equation for the convective biomass transport velocity must be established. This equation is similar to the Euler equations of fluid dynamics. It contains a further unknown quantity to be modeled (in the Euler equations, this is the pressure term). Indeed, this quantity which may be called biomass pressure is responsible for generating the spreading velocity field, just as the pressure drives fluid flow. In the paper of Wood \& Whittaker (1999), the model was closed only in the one-dimensional case like in Wanner \& Gujer (1986) and no model for biomass pressure in the general multi-dimensional case could be given.

\subsection{Formulation of a Density-Dependent Diffusion-Reaction Model for Biomass Spreading}

An alternative to a convective spreading mechanism is spreading due to a diffusive flux. Since diffusion with a constant diffusion coefficient leads to an instantaneous biomass spreading, which contradicts postulate $i$ ), and is able neither to guarantee existence of a bound as required in postulate iii) nor to guarantee $i$ ), a density-dependent diffusion coefficient for biomass must be introduced which vanishes in the liquid region. In order to take the environmental conditions into account which are responsible for the availability of nutrients in the liquid region, the biofilm growth model must include an accurate enough description of transport processes in $\Omega_{1}$, that is hydrodynamics and mass transfer. Thus, the spatio-temporal model should relate the variables

$t \geq 0 \quad$ time as an independent variable

$x \in \Omega \quad$ space coordinate as an independent variable

$m(t, x) \quad$ biomass density as a dependent variable

$c(t, x) \quad$ nutrient concentration as a dependent variable

$u(t, x) \quad$ flow velocity vector in the liquid region as a dependent variable

$p(t, x) \quad$ fluid pressure as a dependent variable

$d_{1,2}(m)$ diffusion coefficients for $c$ and $m$ as variable model parameters

$f(c, m)$ nutrient consumption rate as a variable model parameter

$g(c, m)$ biomass production rate as a variable model parameter

The distinction between liquid region $\Omega_{1}$ and biofilm structure $\Omega_{2}$ is made by biomass density $m(t$, $x)=0$ or $m(t, x)>0$, respectively. The general den- 
sity-dependent diffusion model for biofilm growth is proposed as

$$
\begin{gathered}
\nabla \cdot u=0, \quad \frac{\partial u}{\partial t}+u \cdot \nabla u=-\frac{1}{\rho} \nabla p+\nabla^{2} u \\
\text { in } \Omega_{1}=\{x \in \Omega \mid m(t, x)=0\} \\
u \equiv 0 \text { in } \Omega_{2}=\{x \in \Omega \mid m(t, x)>0\} \\
\frac{\partial c}{\partial t}+u \cdot \nabla c=\nabla \cdot\left(d_{1}(m) \nabla c\right)-f(c, m) \\
\frac{\partial m}{\partial t}=\nabla \cdot\left(d_{2}(m) \nabla m\right)+g(c, m) \\
f(c, m)=\frac{k_{1} c m}{k_{2}+c}, \quad g(c, m)=k_{3}\left(f(c, m)-k_{4} m\right)
\end{gathered}
$$

with boundary conditions for the dependent variables $u, p, c$, and $m$, appropriate to the particular system being considered.

Here, (1) are the continuity and incompressible Navier-Stokes equations describing the fluid flow in the liquid region $\Omega_{1}$, where the density $\rho$ and kinematic viscosity $v$ are constants. Equation (2) describes the transport and consumption of nutrients. In the liquid region $\Omega_{1}$, nutrients are transported by convection and diffusion. In the solid biofilm region $\Omega_{2}$, the transport is diffusive. The diffusion coefficient for nutrients is given by the function $d_{1}(m)>0$. Nutrients are consumed in $\Omega_{2}$ with reaction rate $f(c, m)$ given by (4). This is the standard Monod reaction which is used throughout the biofilm modelling literature. Equations (1) and (2) are derived from first principles. They are well-known and have been studied in the context of mass transfer and conversion in biofilms in two- and three-dimensional systems by Picioreanu $e t$ al. (2000) and Eberl et al. (in press).

Equation (3) is the newly proposed evolution equation for biomass density. Spatial spreading is described by the diffusive flux $d_{2}(m) \nabla m$, with the density-dependent diffusion coefficient $d_{2}(m) \geq 0$. The formation of new biomass is due to the production term $g(c, m)$ given by (4), including a wastage term $-k_{3} k_{4} m$. Since the regions $\Omega_{1}$ and $\Omega_{2}$ depend on $m(t, x)$ and so vary with time, no a priori decoupling of the hydrodynamic model (1) from the evolution equations (2) - (4) is possible. In the nutrient consumption and biomass production and decay terms $f(c, m), g(c, m)$, the parameters $k_{1}, \ldots, k_{4}$ are non-negative and may be regarded as given. We may assume that the nutrient diffusion coefficient $d_{1}(m)$ is positive, bounded and piecewise differentiable. The biomass diffusivity function $d_{2}(m)$ must have a form which predicts that solutions to $(1)-(4)$ satisfy postulates $i$ ) $-v i$ ).

It is known that an exponential ansatz for $d_{2}(m)$ can avoid instantaneous diffusion (Murray, 1993). That is, the diffusive transport mechanism is locally not active as long as $m=0$. This leads to degeneracy of the differential equation as $m=0$. In order to ensure existence of a bound iii), a singularity is introduced when $m=m_{\max }$. Thus, a first suggestion for the density-dependent diffusion coefficient $d_{2}(m)$ is

$$
d_{2}(m)=\left(\frac{\epsilon}{m_{\max }-m}\right)^{a} \cdot m^{b}
$$

This function vanishes for $m=0$ and $d_{2}(m) \approx 0$ as long as $m$ is appreciable smaller than $m_{\text {max }}$. As $m \rightarrow$ $m_{\text {max }}$ it becomes very large and leads to diffusive transport. In (5), the parameter $a$ is to be chosen so as to guarantee $i i i$ ), while $\varepsilon$ and $b$ are responsible for $i$ ) and $i$ i). The postulate $i v)$ is obviously satisfied, since $g(c, m)$ is the only biomass source term in the model. Postulate $v$ ) is satisfied for nutrient transport and consumption (2) and it is satisfied also for the hydrodynamics (1), if a sharp front $i$ ) can be obtained separating the bulk liquid region $\Omega_{1}$ from the solid biomass region $\Omega_{2}$, as in Figure 1 .

The physical interpretation of (3) with (5) is that the biomass diffusivity vanishes as $m$ becomes small but increases as $m$ grows due to biochemical reaction (4). Moreover, for $m \approx 0$, (3) tends to the biomass production equation of classical one-dimensional or multi-dimensional local-rule biofilm models. However, note, that - in contradiction to discrete local rule models - no probabilistic elements are included in the evolution model.

\subsection{First Qualitative Discussion and Model Simplifications}

The complete model (1) - (5) is mathematically rather complicated and in its generality not easily 
accessible for analytical and qualitative interpretation. However, some trivial solutions can be found easily. One such solution has $m \equiv 0$ (no biomass in the system) together with any concentration field $c$ satisfying the linear, transient, homogenous convection-diffusion equation and any flow field $u$ satisfying the Navier -Stokes equations. On the other hand, if there are no nutrients available, the biomass will decay and finally the system converges to the stable steady state $c \equiv 0, m \equiv 0$ for nutrients and biomass together with an appropriate solution of the Navier-Stokes equation. This can be generalized: if there are nutrients in the system initially, but no further nutrients are added, $c$ will tend to 0 due to consumption and the system will again converge to this steady state. Though these solutions describe important physical special cases, they are not very helpful for the description of spatial heterogeneities in biofilm formation. Therefore, for further analysis additional simplifications must be induced into the model.

A major difficulty of the model results from the Navier-Stokes equation (1). Since we are mainly interested in the behaviour of the biomass evolution equation (3) with (5), we will restrict ourselves to the hydrostatic case in this first study, i.e. we assume that $u \equiv 0$. In the presence of a flow field, nutrient concentration boundary layers around the biofilm structure will be thinner and, hence, the nutrient gradients at the fluid/solid transition will be steeper leading to enhanced mass transfer and conversion rates (see Eberl et al, in press).

Many experimental studies have been carried out to determine the nutrient diffusion coefficient $d_{1}(m)$. Depending on the size of molecules, it may differ in the bulk liquid $\Omega_{1}$ and in the biofilm $\Omega_{2}$ while remaining of the same order of magnitude (Bryers \& Drummond, 1998). For small molecules it is almost the same in both regions. Again, since we are focusing on the biomass spreading model (3) with (5), the actual form of the piecewise smooth, positive function $d_{1}(m)$ is not critical and has only minor relevance. Therefore, for simplicity we assume $d_{1}(m) \equiv$ $d_{1}$ to be constant. Introducing dimensionless dependent variables $M:=m / m_{\max }$ and $C:=c / c_{0}$, the simplified model reads

$$
\begin{gathered}
\frac{\partial C}{\partial t}=d_{1} \nabla^{2} C-F(C, M) \\
\frac{\partial M}{\partial t}=m_{m a x}^{b-a} \nabla \cdot\left[\left(\frac{\epsilon}{1-\bar{M}}\right)^{a} M^{b} \nabla M\right]+G(C, M)
\end{gathered}
$$

with

$$
\begin{gathered}
F(C, M)=K_{1} \frac{M C}{K_{2}+C}, \\
G(C, M)=K_{3} \frac{C M}{K_{2}+C}-K_{4} M \\
K_{1}=m_{\text {max }} \frac{k_{1}}{c_{0}}, \quad K_{2}=\frac{k_{2}}{c_{0}}, \\
K_{3}=k_{3} k_{1}, \quad K_{4}=k_{3} k_{4}
\end{gathered}
$$

This system of diffusion-reaction equations for biomass and nutrients resembles a spatio-temporal predator-prey model for biomass and nutrients.

\section{NUMERICAL ILLUSTRATIONS OF MODEL BEHAVIOUR}

Since equations $(6)-(8)$ are still difficult to treat analytically, even though we have neglected the flow field, numerical experiments are carried out to validate the model behaviour. The goal is to show that postulates $i)-v i$ ) are satisfied and that the biofilm structure generated by the model is sensitive to crucial parameters in a manner similar to that observed in a biofilm reactor. Picioreanu et al. (1998b) grouped these parameters into a dimensionless number

$$
\mathcal{G}=l^{2} \frac{\mu_{m} m_{\max }}{d_{1} c_{0}}
$$

In this definition, $l$ is a constant characteristic length and $\mu_{m}$ is the specific growth rate. Since we are interested only in qualitative variations of $\mathcal{G}$ rather than its quantitative value, the particular definition of $l$ is not of relevance here. In our model formulation, $\mu_{m} \quad$ is included in $k_{1}$ through $k_{1}=-m_{\max }\left(\frac{\mu_{m}}{Y_{X S}}+m_{s}\right)$ where $Y_{X S}$ is the substrate growth yield factor and $m_{s}$ is the maintenance coefficient. These biochemical parameters define model parameters $k_{3}=Y_{X S} / m_{\max }$ and $k_{4}=m_{s} m_{\max }$. $k_{2}=K_{s}$ is the Monod saturation constant. 
TABLE I Model parameters used in one-dimensional (1D) and three-dimensional (3D) numerical studies. The symbol $\sim$ represents a value equal to the one in the previous column

\begin{tabular}{cccccccc}
\hline par. & unit & $I D(A)$ & $1 D(B)$ & $I D(C)$ & $1 D(D)$ & $3 D(A)$ & $3 D(B)$ \\
\hline$d_{1}$ & $\mathrm{~m}^{2} / \mathrm{s}$ & $1.6 \cdot 10^{-9}$ & $\sim$ & $\sim$ & $\sim$ & $1.6 \cdot 10^{-9}$ & $\sim$ \\
$\mu_{m}$ & $1 / \mathrm{s}$ & $1.5 \cdot 10^{-5}$ & $\sim$ & $\sim$ & $\sim$ & $1.5 \cdot 10^{-5}$ & $\sim$ \\
$Y_{X S}$ & 1 & 0.045 & $\sim$ & $\sim$ & $\sim$ & 0.045 & $\sim$ \\
$K s$ & $\mathrm{~kg} / \mathrm{m}^{3}$ & $3.5 \cdot 10^{-5}$ & $\sim$ & $\sim$ & $\sim$ & $1.5 \cdot 10^{-5}$ & $\sim$ \\
$m_{s}$ & $1 / \mathrm{s}$ & $3 \cdot 10^{-5}$ & $\sim$ & $\sim$ & $\sim$ & $3 \cdot 10^{-5}$ & $\sim$ \\
$m_{\max }$ & $\mathrm{kg} / \mathrm{m}^{3}$ & 60 & $40 / 80$ & 60 & $60 / 90$ & 40 & $30 / 80$ \\
$c_{0}$ & $\mathrm{~kg} / \mathrm{m}^{3}$ & $4 \cdot 10^{-3}$ & $2 / 4 \cdot 10^{-3}$ & $2 \cdot 10^{-3}$ & $1 / 2 \cdot 10^{-3}$ & $2 \cdot 10^{-3}$ & $2 / 0.6 \cdot 10^{-3}$ \\
$M_{0}$ & 1 & 0.8 & 0.8 & 0.8 & 0.8 & 0.8 & 0.8 \\
$\varepsilon$ & 1 & $10^{-5}$ & $\sim$ & $\sim$ & $\sim$ & $10^{-4}$ & $\sim$ \\
$a$ & 1 & 4 & $\sim$ & $\sim$ & $\sim$ & 4 & $\sim$ \\
$b$ & 1 & 4 & $\sim$ & $\sim$ & $\sim$ & 4 & $\sim$ \\
\hline
\end{tabular}

The numerator in (9) contains the biological factors whereas the denominator contains the local nutrient availability. For large values of $\mathcal{G}$, the regime is transport limited, for low values it is growth limited. In the first case the biomass forms a less regular and rough structure, in the latter it becomes more compact and grows faster (Picioreanu et al., 1998b). In our study, variation of $\mathcal{G}$ arises only from changes of $m_{\max }, d_{1}$, and $c_{0}$. The numerical values of physical and biological model parameters are given in Table I together with the parameters of (5). They are taken from Picioreanu (1999) and have been modified where appropriate in order to obtain varying $\mathcal{G}$ numbers.

The numerical simulation was performed applying finite difference methods. The spatial derivatives have been discretized on a regular grid for both, $C$ and $M$, using a standard centred scheme on the compact stencil. It is well-known that finite difference methods with explicit time-integration suffer from a strict stability restriction for the maximum admissible time-step $\Delta t<\Delta x^{2}$. const, where the constant is the smaller the faster the diffusion process is. This makes explicit methods inefficient for fast diffusion processes. On the other hand, explicit time-stepping on a compact stencil assures that information travels only as far as one grid cell per time-step. This is an interesting property for the spreading of biomass, i.e. the integration of (7). Since $M=0$ holds in $\Omega_{1}$, in an explicit method the only grid cells which need to be considered are those in $\Omega_{2}$ and those of $\Omega_{1}$ for which the compact stencil accesses points of $\Omega_{2}$. This is an important advantage, in particular in the initial stages, when $\Omega_{2}$ is much smaller than $\Omega_{1}$. Therefore, a hybrid time-integration strategy has been applied. The slower biomass spreading process is solved explicitly, whereas the faster nutrient transport process is discretized implicitly. This requires the solution of a semi-linear algebraic system during every time-step for which we used a Newton-BiCGSTAB method. The explicit time-step size was adaptively controlled using as estimate for the nonlinear equation the stability criterion for a linear diffusion equation.

\subsection{One-dimensional Pre-studies}

We first describe some one-dimensional experiments. Of course, these give no information about the formation of locally heterogeneous structures, but they are computationally cheap and allow a first validation of some important features of the model. In particular, we can check postulates $i$ ), ii), iii). Furthermore, even in one-dimensional examples, we can test the model behaviour with regard to simple spatial heterogeneities in the nutrient field and the influence of kinetic model parameters. 
After the simplifications discussed above, the general one-dimensional model equation for $\Omega=[0, L]$ reads

$$
\begin{aligned}
C_{t} & =d_{1} C_{x x}-F(C, M) \\
M_{t} & =\left(D_{2}(M) M_{x}\right)_{x}+G(C, M)
\end{aligned}
$$

with $D_{2}(M)=m_{\max }^{b-a}\left(\frac{\epsilon}{1-M}\right)^{a} M^{b}$. Different experimental arrangements are expressed by varying the initial and boundary conditions for this system of partial differential equations. The length of the system was chosen to be $L=10^{-4} \mathrm{~m}$, divided using 64 equally spaced grid points $\left(\Delta x=1.6 \cdot 10^{-6} \mathrm{~m}\right)$.

\section{(A) growth on a solid surface}

A first example is chosen similar to classical one-dimensional biofilm models. A bacterial inoculum of thickness $2 \Delta x$ is attached to the solid wall at $x=$ 0 and at the other end $x=L$ the nutrient concentration is fixed. Then, initial and boundary conditions are

$$
\begin{aligned}
C_{x}(t, 0) & \equiv 0, \quad C(t, L) \equiv 1 \quad \forall t \geq 0 ; \\
C(0, x) & =1 \quad \forall x \in[0, L] \\
M_{x}(t, 0) & \equiv 0, \quad M_{x}(t, L) \equiv 0 \quad \forall t \geq 0 ; \\
M(0, x) & = \begin{cases}M_{0} & \text { for } x \leq 2 \Delta x \\
0 & \text { for } x>2 \Delta x\end{cases}
\end{aligned}
$$

A typical result is shown in Figure 2. Here and in all subsequent model studies we observe the formation of a sharp front separating the liquid from the solid regions (i.e. postulate $i$ ) together with the waiting time behaviour $i i)$ ) and the existence of a biomass density bounds iii). Since characteristic time-scales for nutrient transport and consumption are much smaller than the characteristic time-scales for biomass formation (Kissel et al., 1984) and since the biomass density itself is not uniform at $t=0$, the initially uniform nutrient concentration is disturbed immediately and a non-uniform nutrient concentration field develops. In the biofilm at $x=0, C$ tends rapidly to 0 .

\section{(B) one-dimensional growth with asymmetric boundary conditions}

The next case is to demonstrate how environmental conditions and biological parameters - i.e. the number

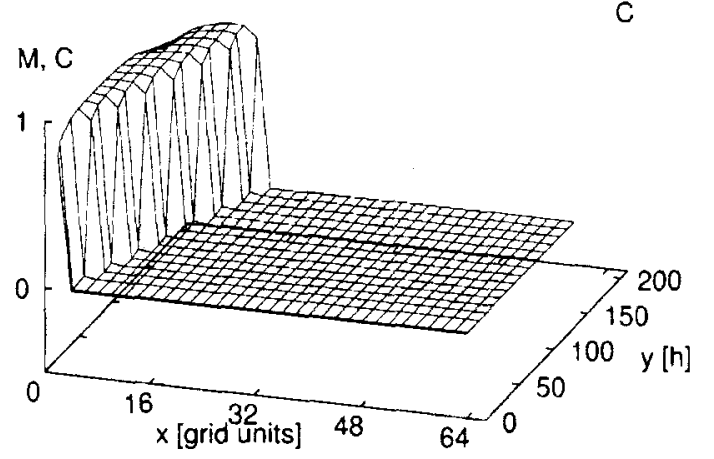

FIGURE 2 Growth of a bacterial colony attached to a solid surface Shown are biomass density $M$ and nutrient concentration $C$. Nutrients are consumed in the biomass region. The biomass forms a sharp front and spreads only for biomass densities $M \simeq M_{\max }$

$\mathcal{G}$ as defined in (9) - affect biomass growth and spreading in our model description. For this purpose, an inoculum biomass covering a small interval of length $2 \Delta x$ was placed approximately in the middle of the computational domain $[0, L]$ and nutrients were fed at one end only. Hence, we have

$$
\begin{gathered}
C_{x}(t, 0) \equiv 0, \quad C(t, L) \equiv 1 \quad \forall t \geq 0 ; \\
C(0, x)=1 \quad \forall x \in[0, L] \\
M_{x}(t, 0) \equiv 0, \quad M_{x}(t, L) \equiv 0 \quad \forall t \geq 0 ; \\
M(0, x)= \begin{cases}M_{0} & \text { for } x \in\left[\frac{L}{2}-\Delta x, \frac{L}{2}+\Delta x\right] \\
0 & \text { elsewhere }\end{cases}
\end{gathered}
$$

This example is more complicated than the standard one-dimensional model because now biomass spreads in two directions, whereas an adhesion of biomass to a solid surface as in (A) allows movement only in one direction, as in the spreading mechanism of the classical one-dimensional model of Wanner \& Gujer (1986).

Starting from a reference state (Figure $3 a$ ), first the maximum biomass density has been decreased (Figure $3 b$ ) and then the nutrient concentration was increased (Figure 3c). Both b) and c) of this example result in a value $\mathcal{G}$ lower than in a). Since different boundary conditions for $C$ are specified at the two ends of the computational domain, the initially uni- 

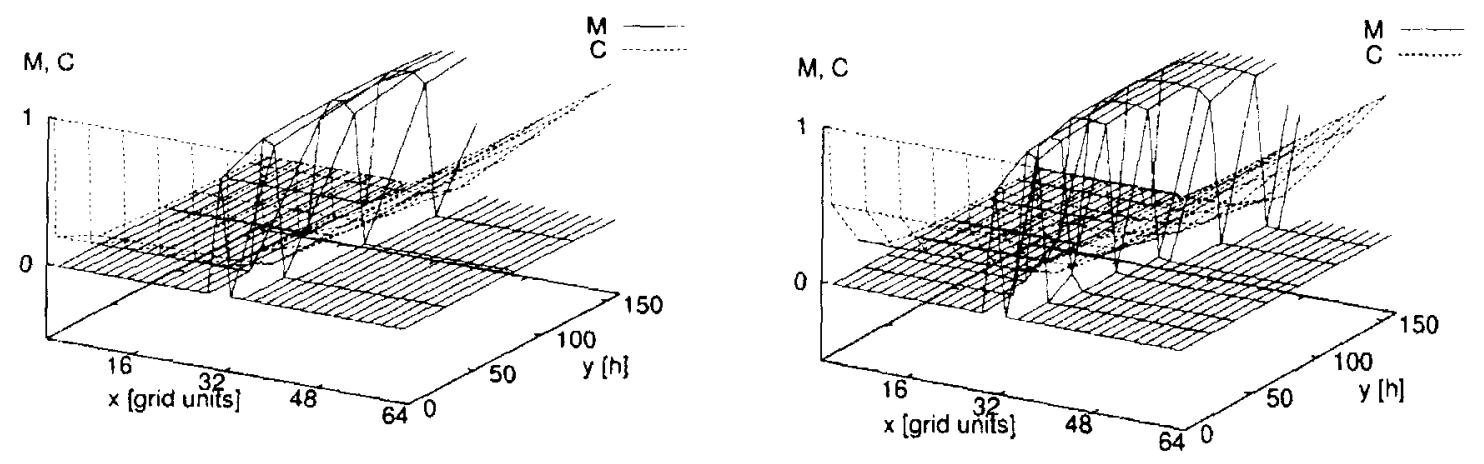

a)

b)

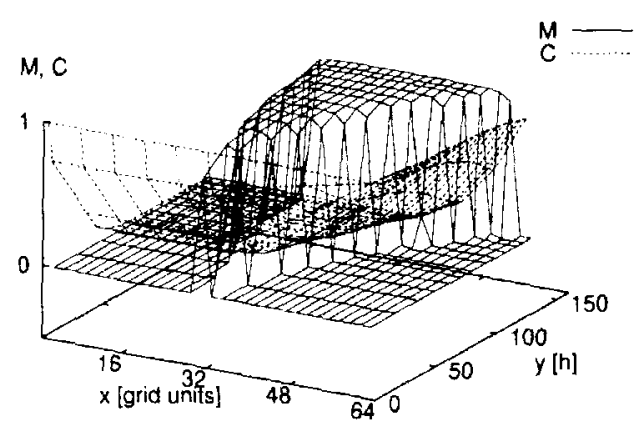

c)

FIGURE 3 Growth of a bacterial colony in the centre of the one-dimensional domain for varying environmental conditions and different biomass bounds: Shown are biomass density $M$ and nutrient concentration $C$ : Nutrients are depleted from right to left and the biofilm structure develops different on both directions, due to different boundary conditions at $x=0$ and $x=L$. a) Starting from parameter set $\operatorname{mmax}=80 \mathrm{~kg} / \mathrm{m}^{3}, c_{0}=2 \mathrm{~g} / \mathrm{m}^{3}$, biomass growth and spreading is accelerated $\left.\mathrm{b}\right)$ after $\mathrm{m}_{\max }$ is decreased $\left(\mathrm{m}_{\max }=40 \mathrm{~kg} / \mathrm{m}^{3}, \mathrm{c}_{0}=2 \mathrm{~g} / \mathrm{m}^{3}\right)$, or $\mathrm{c}$ ) after $c_{0}$ is increased $\left(c_{0}=4 \mathrm{~g} / \mathrm{m}^{3}, \operatorname{mmax}=40 \mathrm{~kg} / \mathrm{m}^{3}\right) . \mathcal{G}$ decreases from a) through c)

form nutrient field soon becomes spatially heterogeneous. After a short initial phase, virtually no nutrient remains on the side of the biofilm away from the source in a) and b), due to nutrient consumption in the solid region. Therefore, biomass grows only towards the nutrient source. Only in case c) an appreciable quantity of nutrients remains on this side and the biomass spreads in both directions; that is, the developing biofilm structure shows the same heterogeneity as the nutrient field. As expected, the changes b) and c) accelerated the formation of the biofilm; in the first case because more nutrients are available, in the second case because the spreading mechanism acts sooner (i.e. for smaller $M$ ). In all cases, due to lack of nutrients the decay term dominates the growth term in $G(C, M)$ on the shielded side and biomass depletion starts. 


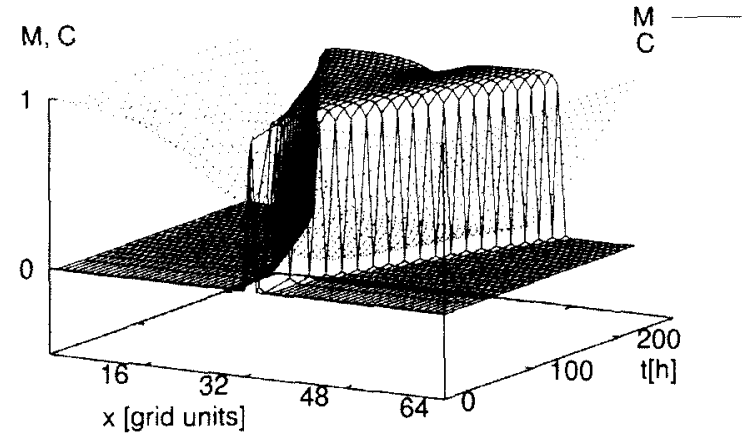

FIGURE 4 Biomass growth and spreading under symmetric boundary conditions. Shown are biomass density $M$ and nutrient concentration C. Biomass grows symmetrically. Nutrients are consumed in the structure. At a given moment biomass starts to decay in the centre due to nutrient limitation

\section{(C) one-dimensional growth with symmetric initial and boundary conditions}

While the last example (B) already contains a simple spatial heterogeneity - though only in one dimension - we will now generate a symmetrical setup to evaluate how a biofilm structure may develop in a regular nutrient field. The initial and boundary conditions in this case read

$$
\begin{gathered}
C(t, 0) \equiv 1, \quad C(t, L) \equiv 1 \quad \forall t \geq 0 ; \\
C(0, x)=1 \quad \forall x \in[0, L] \\
M_{x}(t, 0) \equiv 0, \quad M_{x}(t, L) \equiv 0 \quad \forall t \geq 0 ; \\
M(0, x)= \begin{cases}M_{0} & \text { for } x \in\left[\frac{L-\Delta x}{2}, \frac{L+\Delta x}{2}\right] \\
0 & \text { elsewhere }\end{cases}
\end{gathered}
$$

According to vi) we expect a symmetrically developing biofilm structure. Figure 4 refers to this case and clearly shows that our expectations are fulfilled. The biofilm grows symmetrically in both directions, i.e. towards both nutrient sources. After some time, when the structure is so thick that, due to consumption, nutrients are depleted near its center and decay starts and the biomass density decreases again.

\section{(D) One-dimensional growth with merging colonies under symmetric initial and boundary conditions}

As a final one-dimensional example, the collision of two sharp biomass fronts is investigated. For this pur- pose we place two equally sized colonies on the interval $[0, L]$ and apply the same boundary conditions as in $(\mathrm{C})$ :

$$
\begin{gathered}
C(t, 0) \equiv 1, \quad C(t, L) \equiv 1 \quad \forall t \geq 0 \\
C(0, x)=1 \quad \forall x \in[0, L] \\
M_{x}(t, 0) \equiv 0, \quad M_{x}(t, L) \equiv 0 \quad \forall t \geq 0 ; \\
M(0, x)= \begin{cases}M_{0} & \text { for } x \in\left[x_{1}, x_{2}\right] \cup\left[x_{3}, x_{4}\right] \\
0 & \text { elsewhere }\end{cases}
\end{gathered}
$$

with $x_{1}=L-x_{4}, x_{2}=L-x_{3}, 0<x_{2}-x_{1}=x_{4}-$ $x_{3}=\Delta x \ll L$

This case appears critical, since in the collision two moving interfaces may turn into interior points, then allowing mass transfer from one colony to the other. With respect to spatial modelling this is a very important case since in general the inoculum will be distributed in small colonies over the whole substratum rather than being concentrated in a single colony.

Figure 5a, shows that our newly formulated model is able to describe the merging of colonies while satisfying the biomass density bound postulation $i i i$ ). Biomass spreads in both directions from each initial colony. Figure $5 \mathrm{~b}$ shows the same experiment, but with increased $m_{\max }$ and decreased $c_{0}$ (i.e. increased $\mathcal{G})$. There, delayed and slower biomass spreading (compared to Figure 5a) is observed. Between the two initial colonies nutrients become limited, so consequently no merging takes place but instead a channel remains separating the two biomass clusters.

\subsection{Discussion of One-dimensional Examples}

The one-dimensional results (A), (B), (C), (D) allow preliminary deductions of the model behaviour in the presence of spatial heterogeneities in a biofilm structure. Increased nutrient availability or decreased maximum biomass concentration (i.e. decreased $\mathcal{G}$ ) accelerate the spatial spreading of biomass and, in consequence, colonies merge earlier and form a more compact spatial structure. In contrast, increase in $\mathcal{G}$ might keep colonies isolated as a consequence of delayed biomass spreading and lack of nutrients. If the nutrient availability is only locally increased (i.e. 


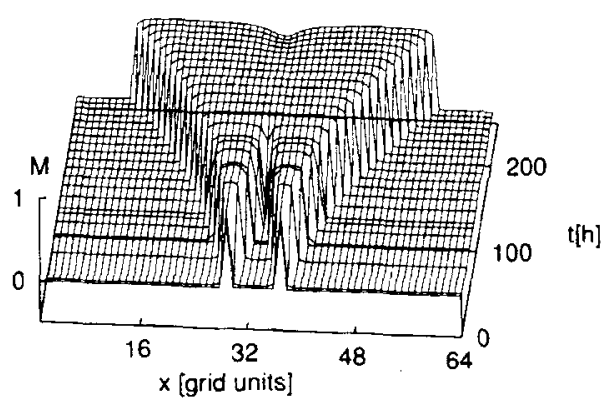

a)

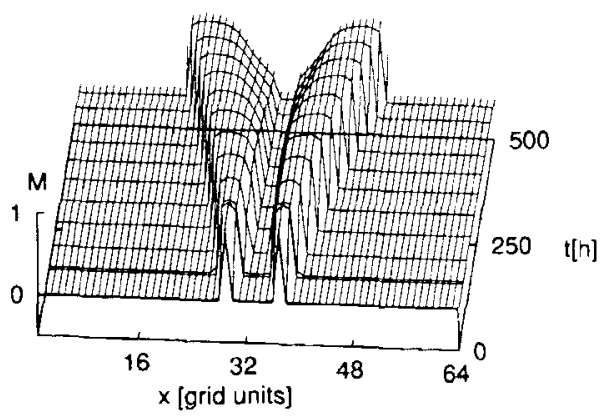

b)

FIGURE 5 Spreading of biomass with two initial colonies under symmetric boundary conditions for different $\mathcal{G}$ : Shown is biomass density $M$. In a) a compact structure develops after merging of both colonies $\left(\mathrm{m}_{\mathrm{m}} \mathrm{ax}=60 \mathrm{~kg} / \mathrm{m}^{3}, \mathrm{c}_{0}=2 \mathrm{~g} / \mathrm{m}^{3}\right)$ while in b) the two colonies remain separated $\left(\mathrm{m}_{\mathrm{m}} \mathrm{ax}=90 \mathrm{~kg} / \mathrm{m}^{3}, \mathrm{c}_{0}=1 \mathrm{~g} / \mathrm{m}^{3}\right)$, due to an increase in $\mathcal{G}$

the nutrient field is spatially irregular), biomass production may also increase only locally and a spatially irregular structure may develop. A symmetric inoculum under symmetric boundary conditions will develop into a symmetric, but not necessarily compact, structure, whereas the biomass spreads in an asymmetric, but not necessarily non-compact, fashion if the initial and/or boundary conditions are already spatially heterogenous. Even if the initial nutrient field is uniform, it will become heterogeneous if the biomass distribution (and, hence, nutrient consumption) is heterogeneous. The consequent spatially heterogeneous nutrient availability then influences further biomass production in turn. Thus, we have seen that the proposed model is capable of describing cluster-and-channel biofilms which are observed in numerous laboratory experiments. This will be verified in the sequel by means of fully three-dimensional simulation.

\subsection{Three-dimensional Studies: the Fully Spatially Heterogeneous Case}

After one-dimensional analysis revealed that the proposed model satisfies postulates $i$ ) $-v i$ ), fully heterogenous three-dimensional computer simulations were carried out to investigate the model behaviour in a general and physically relevant case. For this purpose, the three-dimensional equations (6) and (7) were solved.

A constant nutrient concentration is maintained at the top of the rectangular computational domain $\Omega=\left[0, L_{1}\right] \times\left[0, L_{2}\right] \times\left[0, L_{3}\right]$, i.e. at $x_{3}=L_{3}$. At all other boundaries of $\Omega$ we apply standard outflow boundary conditions. That is, there is no diffusive flux. The initial colonization of the domain (i.e. $\Omega_{2}$ at $t=0$ ) is generated randomly after specification of inoculum density. Together, we have initial and boundary conditions

$$
\begin{gathered}
\frac{\partial C}{\partial n} L_{\partial \Omega \backslash\left\{x_{3}=L_{3}\right\}} \equiv 0, \quad C{\left\lfloor x_{3}=L_{3} \equiv 1\right.} \quad \forall t>0, \\
C(0, x) \equiv 1 \quad \forall x \in \Omega \\
\frac{\partial M}{\partial n}\left\lfloor_{\partial \Omega} \equiv 0, \quad M(0, x)= \begin{cases}M_{0} & \text { for } x \in \Omega_{2} \\
0 & \text { for } x \in \Omega_{1}\end{cases} \right.
\end{gathered}
$$

where $n$ is the outward normal unit vector of the boundary of the computational domain. With the specified Dirichlet boundary conditions for $x_{1}$ and $x_{2}$, the simulated system can be considered a small section of a bigger biofilm system. All simulations presented here were carried out on the equidistant regular grid with spatial step-size $\Delta x=1.89 \mu m$.

\section{(A) development of a spatially heterogeneous biofilm structure}

In a first experiment, a substratum of $64 \times 64$ grid points was inoculated with biomass in 162 grid cells. 

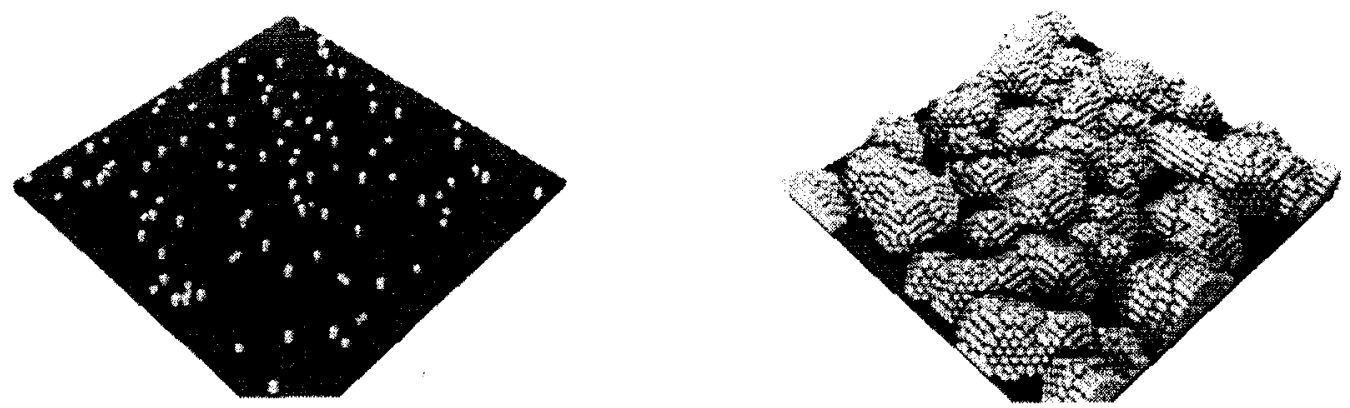

(a) $t=0$

(b) $t=122 h$
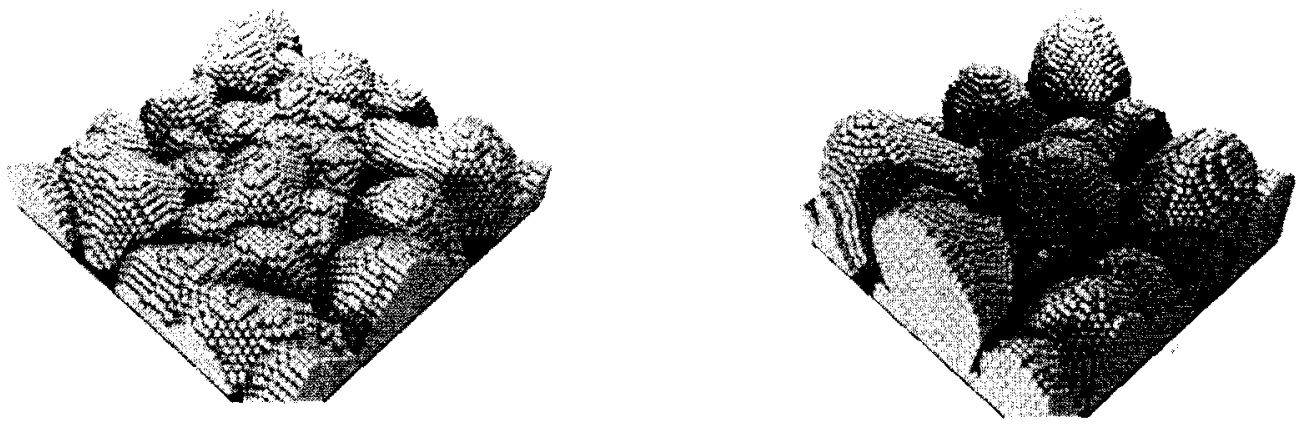

(c) $t=235 h$

(d) $t=445 h$
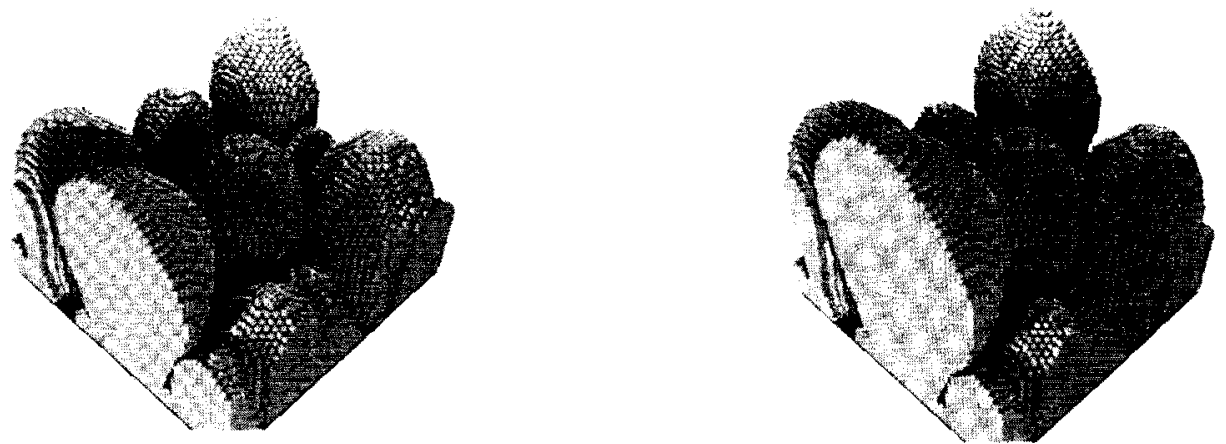

(e) $t=567 \mathrm{~h}$

(f) $t=643 h$

FIGURE 6 Development of a biofilm structure in time: first a wavy structure forms, later when nutrients are limited bigger colonies start to dominate over smaller ones. As a consequence of this competition for nutrients, the bigger colonies grow faster and develop into mushroom shapes while the smaller ones grow much slower

Maximum colony height in the inoculum was two grid cells. The total height of the computational domain is 63 grid cells. The simulation is visualized in Figure 6. First, so long as nutrients are available 
everywhere in the system, the biomass spreads in all directions and forms a wavy layer. With increasing biomass in the system, the nutrient concentration decreases due to increased consumption and finally the nutrients become limited. Then, larger bacterial colonies start to dominate over their smaller neighbours due to higher nutrient consumption. This leads to the formation of mushroom-type structures which are also reported in the experimental literature (e.g. Costerton et al., 1995).

\section{(B) simulation with different inoculum density and varying $\mathcal{G}$}

In a second experiment, the sensitivity of the model to inoculum density and to $\mathcal{G}$ is validated. The system size is $128 \times 64 \times 63$ grid cells. Three initial states were created with 83,411 , and 821 grid cells occupied by biomass. Simulation runs were stopped, after the biofilm reached a maximum height $36 \mu m\left(=0.3 L_{3}\right)$. Afterwards the experiment was repeated with higher maximum biomass and lower bulk nutrient concentration, causing $\mathcal{G}$ to increase by a factor 8.89 .

The results are shown in Figure 7. Independently of the inoculum density it is observed that, for the larger $\mathcal{G}$, the bacterial colonies hardly merge at this stage of biofilm formation. They grow upwards, where nutrients are abundant, rather than spreading horizontally where they are limited. This tendency to form isolated colonies for larger values of $\mathcal{G}$ was already seen in the one-dimensional studies (Figure 5b) and is in good agreement with empirical expectations. For lower $\mathcal{G}$ the behaviour is different. Since nutrients are not limited, biomass spreading is not only towards the source but also horizontally so that colonies may merge. We deduce that the more dense the inoculum, the more compact is the biofilm in the case of lower $\mathcal{G}$, whereas for high $\mathcal{G}$ even for dense inoculum rough and irregular structures might develop. This is in accordance with the findings of Picioreanu et al., 1998b.

\subsection{Discussion of Fully Three-Dimensional Simulations}

Non-uniform initial distribution of biomass disturbs a uniform initial distribution of nutrients so that local nutrient availability soon becomes heterogeneous. With heterogeneous nutrient availability, biomass production becomes non-uniform and spatially heterogeneous biofilm structures develop. The formation of cluster-and-channel biofilm structures is observed with mushroom-shaped colonies dominating over smaller neighbor colonies. Those findings are in good agreement with experimental experience (e.g. Costerton et al., 1995).

In this first study, only biofilm formation under hydrostatic conditions with fixed nutrient concentration at a specified height was investigated. In the hydrodynamic case, the flow field contributes to convective transport of nutrients and, hence, influences their local availability and as a direct consequence influences the biofilm structure. Though already included in the original model formulation (1) - (5) and conceptually straightforward, it is technically very difficult and computationally very expensive to consider hydrodynamics in computer simulations. This is beyond the scope of this first presentation of the new biomass spreading mechanism. Another phenomenon occurring in biofilm systems with flowing bulk liquid is biomass detachment due to shear stresses. This process is not included in the present model formulation (1) - (5) but a model extension is required, as presented by Picioreanu et al. (1999) for a two-dimensional discrete biofilm growth model.

\section{CONCLUSION}

A new spatio-temporal continuum model for biofilm formation is developed. It yields the observation that spatial heterogeneities in the biofilm structure evolve, due to spatial heterogeneities in the environmental conditions. In contrast to previous models it is purely deterministic, yet nevertheless is able to predict spatially highly irregular biofilm formation. The starting point for the model development was the a priori postulation of some required properties of the model. This suggested a quasilinear system of diffusion-reaction equations for biomass and nutrient substrate which can be interpreted as a spatio-temporal predator-prey-model. The diffusivity for biomass spreading 


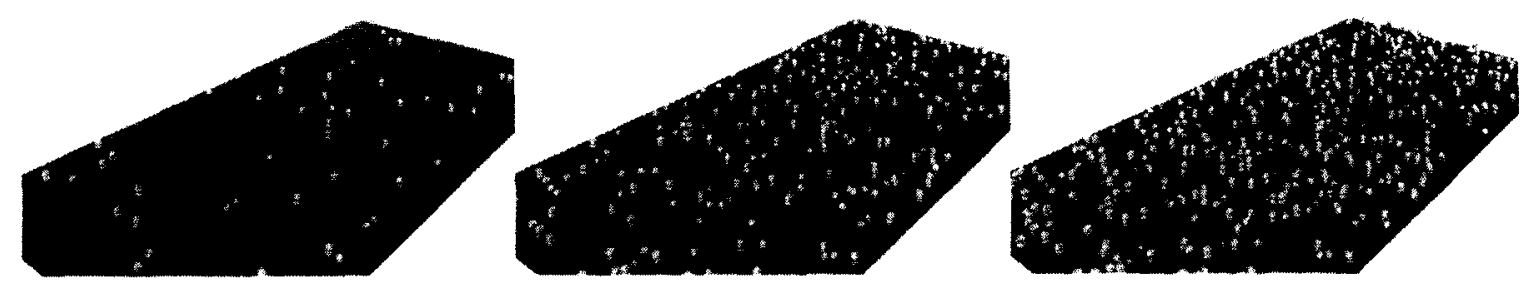

inoculum: 83 grid cells $\quad 411$ grid cells $\quad 821$ grid cells

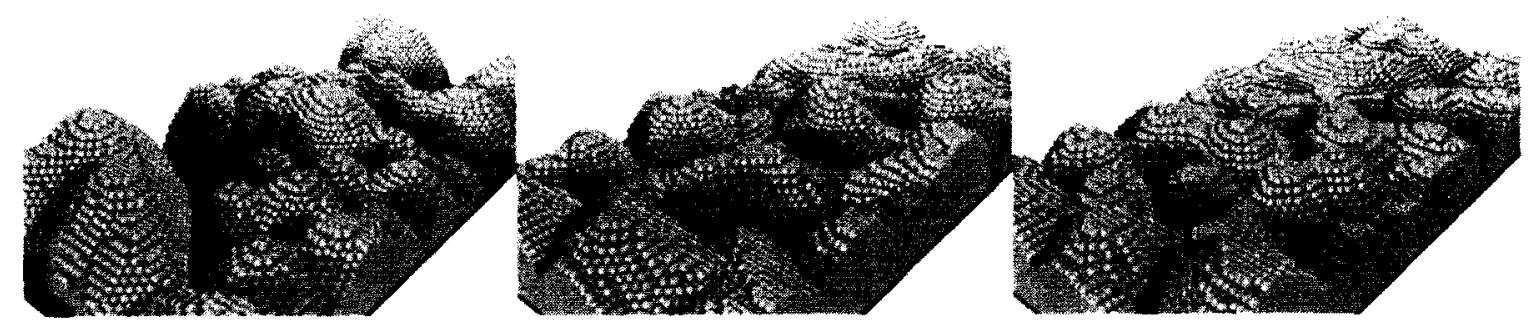

low G-number

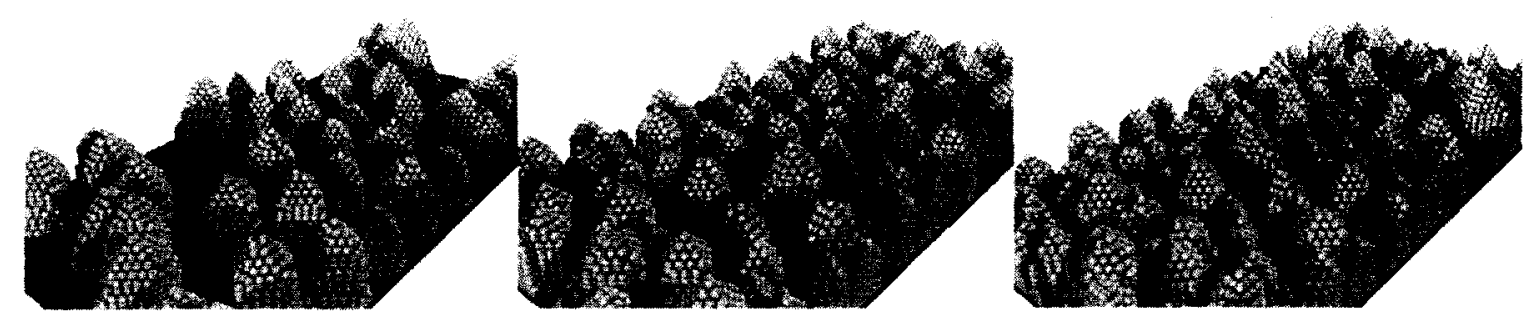

high G-number

FIGURE 7 Biofilm formation for different inoculum densities: for lower $\mathcal{G}$ bacterial colonies merge and form a compact biofilm, for higher $\mathcal{G}$ colonies remain isolated independent of the inoculum density

vanishes as biomass vanishes and has a singularity for maximum biomass density. Analytical progress is very difficult, so model validation was performed by one- and three-dimensional computations. The model behaviour is found to be qualitatively in good agreement with previous experimental and modelling experience. By its construction, the model is invariant to changes of the coordinate system. The biomass density is a continuous function, governed by a differen- tial equation. Possible grid refinements are an issue for the numerical discretization and not of the model itself. Thus, some major previously mentioned drawbacks of the discrete local-rule based approaches are avoided. The model presented in this paper should be considered the first step in deterministic continuum modelling of spatio-temporal irregular biofilm structure. Future steps are the extension for multi-species/multi-substrate biofilms, in order to allow the 
investigation of biofilm formation and reformation as a consequence of an antibiotic therapy. Some further planned extensions are the inclusion of additional processes like biomass detachment and EPS formation.

\section{Acknowledgements}

This study was financially supported by the European Commission under grant number ERBFM RX-CI 970114 (TMR Network BioToBio) and grant number ERBFM GE-CT 95-0051 (the TRACS programme at EPCC). Three-dimensional computations were carried out with the CRAY T3Es of the Edinburgh Parallel Computing Center (EPCC) and the Center for High Performance applied Computing at Delft UT $(\mathrm{HP} \alpha \mathrm{C})$. HJE wants to thank Douglas A Smith (EPCC) for many valuable suggestions on implementational issues.

\section{References}

Bryers JD, Drummond F (1998). Local macromolecule diffusion coeffiecients in structurally non-uniform bacterial biofilms using fluorescence recovery after photobleaching (FRAP), Biotechnology and Bioengineering, 60(4):462-473.

Chaudhry MAS, Beg SA (1998). A Review on the Mathematical Modeling of Biofilm Processes: Advances in Fundamentals of Biofilm Modelling, Chemical Engineering Technology, 21(9):701-710.

Costerton JW, Lewandowski Z, Caldwell DE, Korber DR, Lappin-Scott HM (1995). Microbial Biofilms. Annual Review Microbiology, 49:711-745.

Costerton JW, Stewart PS, Greenberg EP (1999). Bacterial Biofilms: A Common Cause of Persistent Infections, Science, 284:1318-1322

Eberl HJ, Picioreanu C, van Loosdrecht MCM (in press). A three-dimensional numerical study on the correlation of geometrical structure, hydrodynamic conditions, and mass transfer and conversion in biofilms. to appear in Chemical Engineering Science.
Gjalterna A, Arts PAM, van Loosdrecht MCM, Kuenen JG, Heijnen JJ (1994). Heterogeneity of Biofilms in Rotating Annular Reactor: Occurence, Structure, and Consequences. Biotechnology and Bioengineering, 44:194-204.

Hermanowicz SW (1999). Two-dimensional simulations of biofilm development: effects of external environmental conditions. Water Science and Technology, 39(7):107-114

Kayser FH, Bienz KA, Eckert J, Lindemann J (1993). Medizinische Mikrobiologie, 8th edn, Georg Thieme Verlag Stuttgart.

Kissel JC, McCarty PL, Street RL (1984). Numerical Simulation of Mixed-Culture Biofilm, Journal of Environmental Engineering, 110(2):393-411.

van Loosdrecht MCM, Eikelboom D, Gjaltema A, Mulder A, Tijhuis L, Heijnen JJ (1995). Biofilm Structures, Water Science and Technology, 32(8):35-43.

Murray JD (1993). Mathematical Biology, 2nd edn, Springer

Van Loosdrecht MCM, Picioreanu C, Heijnen JJ (1997). A more unifying hypothesis for biofilm structures. FEMS Microbiology Ecology, 24:181-183.

Noguera DR, Pizarro G, Stahl DA, Rittmann BE (1999). Simulation of multispecies biofilm development in three dimensions. Water Science and Technology, 39(7):123-130.

Picioreanu C, van Loosdrecht MCM, Heijnen JJ (1998a). A new combined differential-discrete cellular automaton approach for biofilm modelling: Application for growth in gel beads, Biotechnology and Bioengineering, 57(6):718-731.

Picioreanu C, van Loosdrecht MCM, Heijnen JJ (1998b). Mathematical Modelling of Biofilm Structure with a Hybrid Differential-Discrete Cellular Automaton Approach, Biotechnology and Bioengineering, 58(1):101-116.

Picioreanu C (1999). Multidimensional modeling of biofilm structure, $\mathrm{PhD}$-Thesis, Delft UT.

Picioreanu C, van Lossdrecht MCM, Heijnen JJ (2000) A theoretical study on the effect of surface roughness on mass transport and transformation in biofilms. Biotechnology and Bioengineering, 68(4):355-369.

Rittmann BE, McCarty PL (1980), Model of Steady-State-Biofilm Kinetics. Biotechnology and Bioengineering, 22:2343-2357.

Rittmann BE, Pettis M, Reeves HW, Stahl DA (1999). How biofilm clusters affect substrate flux and ecological selection. Water Science \& Technology, 39(7):99-105.

Wanner O, Gujer W (1986). A Multispecies Biofilm Model. Biotechnology and Bioengineering, 28:314-328.

Wimpenny JWT, Colasanti R (1997). A unifying hypothesis for the structure of microbial biofilms based on cellular automaton models, FEMS Microbiology Ecology, 22:1-16.

Wood BD, Whitaker S (1999). Cellular Growth in Biofilms. Biotechnology and Bioengineering, 64(6):656-669. 


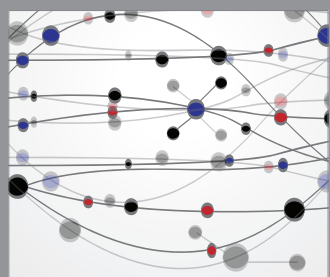

The Scientific World Journal
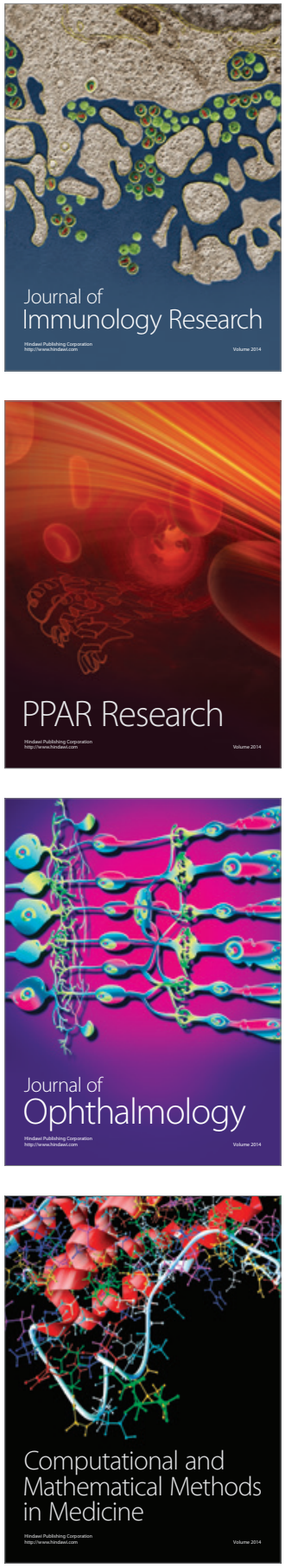

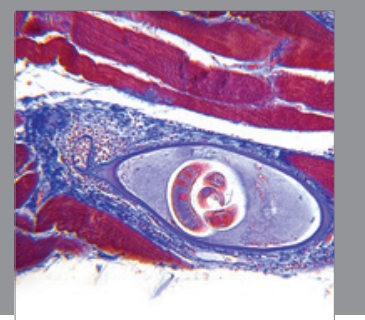

Gastroenterology

Research and Practice
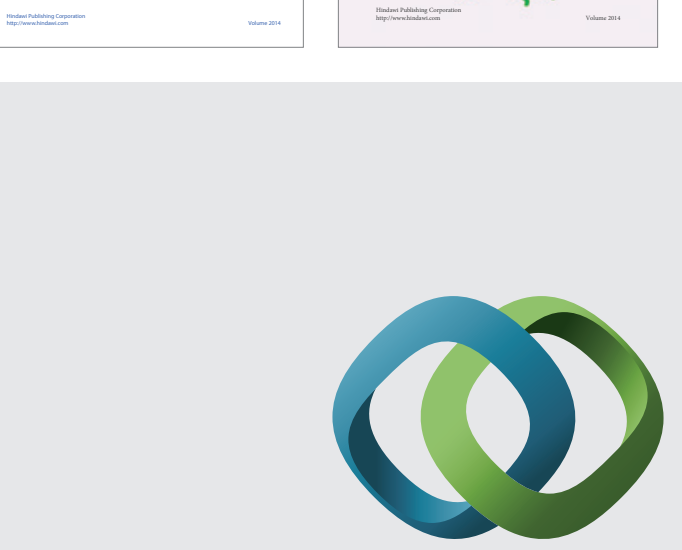

\section{Hindawi}

Submit your manuscripts at

http://www.hindawi.com
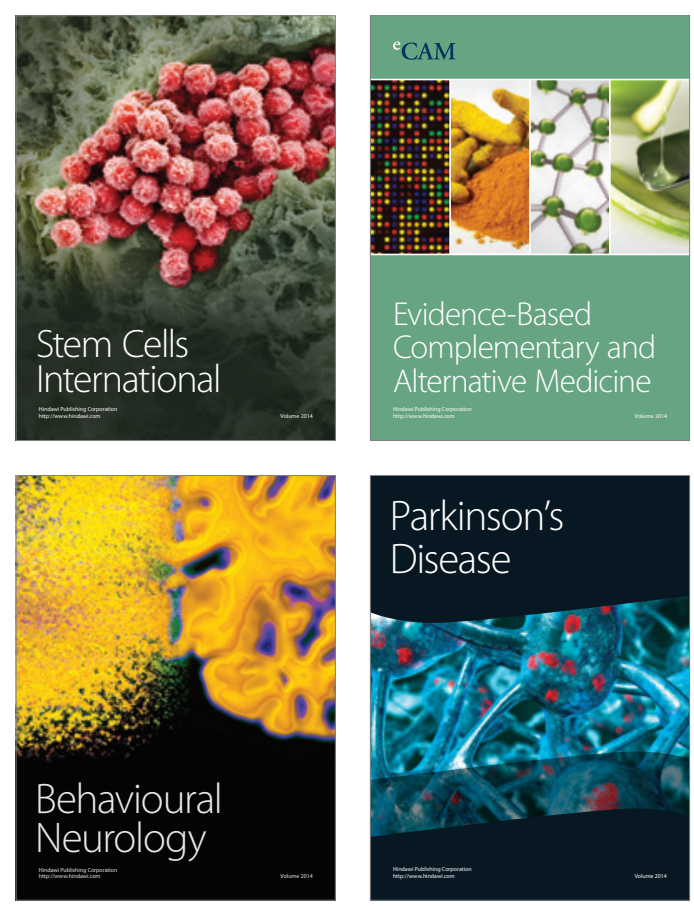

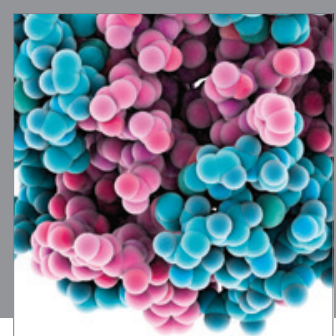

Journal of
Diabetes Research

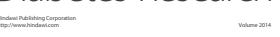

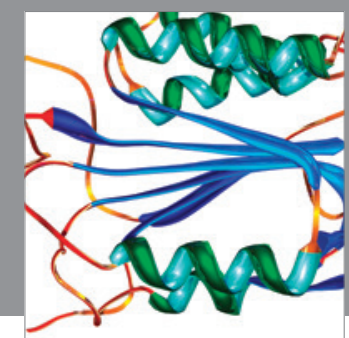

Disease Markers
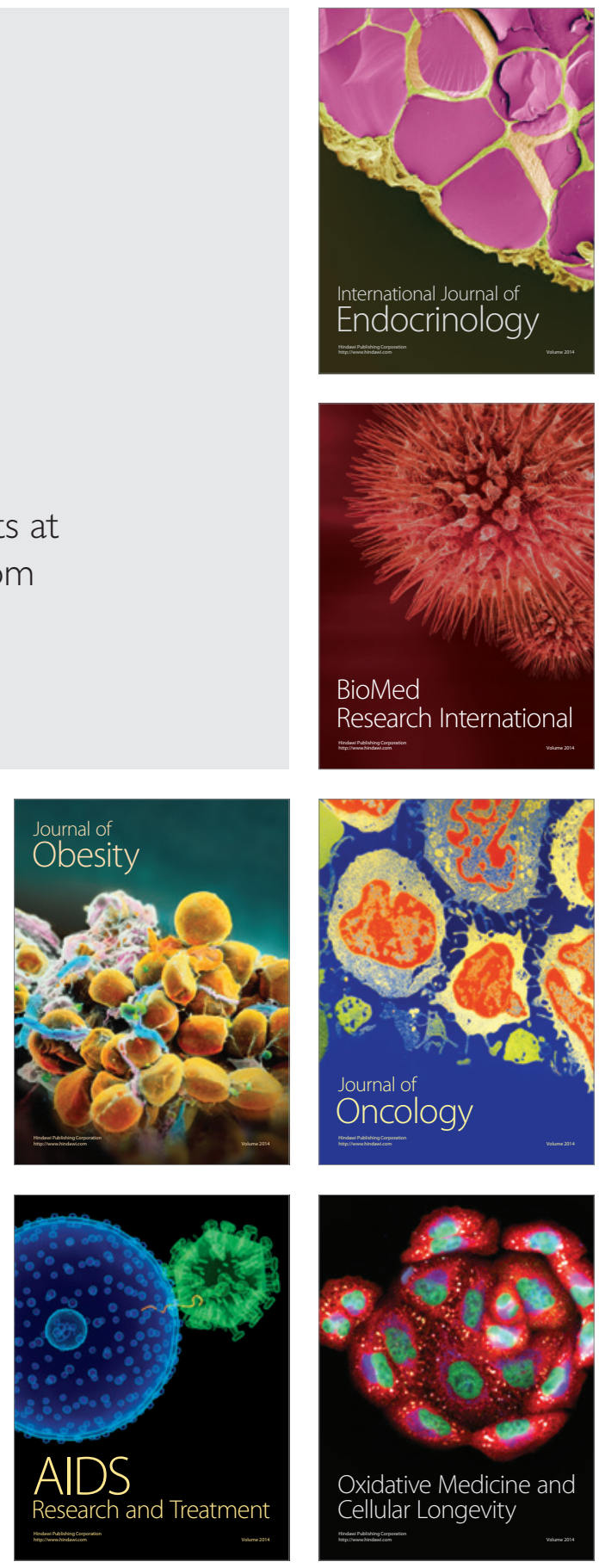